\title{
Sliding charge-density wave in two-dimensional rare-earth tellurides
}

\author{
A. A. Sinchenko \\ National Research Nuclear University (MEPhI), 115409 Moscow, Russia \\ P. Lejay and P. Monceau \\ Institut Néel, CNRS and Université Joseph Fourier, BP 166, 38042 Grenoble, France \\ (Received 30 March 2012; published 25 June 2012)
}

\begin{abstract}
Nonlinear transport properties are reported in the layered DyTe ${ }_{3}$ compound at a temperature below the charge-density-wave (CDW) transition $T_{P}=302 \mathrm{~K}$. Conductivity increases sharply above the threshold electric field. Under application of a rf field, Shapiro steps are clearly observed. These features demonstrate CDW sliding in two-dimensional compounds.
\end{abstract}

DOI: 10.1103/PhysRevB.85.241104

PACS number(s): 71.45.Lr, 61.44.Fw, 72.15.Nj

Interaction between pairs of quasiparticles often leads to broken-symmetry ground states in solids. Typical examples are the formation of Cooper pairs in superconductors, and charge-density waves (CDWs) and spin-density waves (SDWs) driven by electron-phonon and electron-electron interactions, respectively. ${ }^{1}$ Density-wave formation is favored by the nesting of a parallel Fermi surface (FS) at $+k_{F}$ and $-k_{F}$. The first canonical example was demonstrated 50 years ago when the antiferromagnetism in $\mathrm{Cr}$ was identified as an incommensurate SDW transition ${ }^{2}$ resulting from the nesting of electron-hole pocket sheets having a similar shape ${ }^{3}$ (for a review, see also Refs. 4 and 5).

A CDW ground state is characterized by a concomitant spatial modulation $\sim \cos (Q x+\varphi)$ of the electron density and a periodic lattice distortion with the same $Q_{\mathrm{CDW}}=2 k_{F}$ wave vector inducing the opening of a gap in the electron spectrum. The coupled electron-phonon which leads to the Kohn anomaly at high temperature is split into two different modes below the Peierls transition: an optical mode (called amplitudon) and an acoustic phase mode, or phason. ${ }^{1}$

For ideal one-dimensional (1D) conductors, the phase mode exists at zero frequency at $Q_{\mathrm{CDW}}$, realizing the Goldstone mode in systems with spontaneously broken continuous symmetry. Then, a persistent current is provided by the sliding motion of the CDW. ${ }^{6}$ However, various mechanisms such as impurities, interchain interactions, or commensurability pin the phase of the CDW and introduce a gap in the excitation of the phase mode which prevents dc Fröhlich conductivity but leads to large low frequency ac conductivity. The pinning energy must be overcome to initiate the CDW sliding; that can be achieved by the application of an electric field of sufficient strength. ${ }^{1}$ In addition to the increase of conductivity associated with collective CDW motion, above a threshold electric field $E_{T}$ a periodic time dependent voltage is generated, as well as broadband noise. ${ }^{1}$ Sliding CDW properties have been observed in inorganic $\mathrm{NbSe}_{3}, \mathrm{TaS}_{3}, \mathrm{~K}_{0.3} \mathrm{MoO}_{3}$, and $\left(\mathrm{TaSe}_{4}\right)_{2} \mathrm{I}$, as well as in organic (TTF-TCNQ), (TMTSF $)_{2} X$, (Per $)_{2} \mathrm{M}(\mathrm{Mnt})_{2}$, and (fluoranthene $)_{2} X$ one-dimensional compounds.

For systems with strong electronic correlations a wide variety of properties due to strong coupling competition among spin, charge, and lattice degrees of freedom is expected. One fingerprint of the resulting ground states is often phase separation having the form of stripes. Charge order in two-dimensional (2D) organic compounds reveals such an inhomogeneous ground state. ${ }^{8}$ Phase separation is demonstrated excellently in underdoped perovskite oxides, leading to high temperature superconductivity (HTSC), and in manganites in the colossal magnetoresistance regime ${ }^{9}$ in which charge carriers in the $\mathrm{CuO}_{2}$ layers segregate into such stripes that alternate with intermediate spin stripes (for a recent review, see Ref. 10).

Attempts to detect the sliding effect in 2D systems have been, up to now, unsuccessful. Thus, the absence of nonlinearity in current-voltage characteristics (IVCs) was reported in $2 \mathrm{H}-\mathrm{TaSe}_{2}$ and $1 \mathrm{~T}-\mathrm{TaS}_{2}$ up to electric fields of 1 and $10 \mathrm{~V} / \mathrm{cm}$, respectively. ${ }^{11}$ These values are much higher that typical $E_{T}$ in one-dimensional (1D) compounds. One possible reason might be the strong pinning resulting from the triple- $\mathbf{Q}$ structure with three wave vectors of equal amplitude, $120^{\circ}$ apart.

Sliding properties were also searched for in other 2D structures. Thus, 2D quantum systems of electrons of extremely low disorder in GaAs/AlGaAs heterojunctions were shown to exhibit conduction typical of pinned CDWs. ${ }^{12}$ Microwave resonance in conductivity was understood as the pinning mode of the electron or charge ordered crystal. ${ }^{13,14}$ For reentrant integer quantum Hall states, a sharp threshold and a periodic voltage associated with broadband noise were measured. ${ }^{15}$ Although such effects are very similar to the characteristic features of CDW sliding, the very low frequency of the ac voltage generated in the nonlinear state disregards the analogy with a simple CDW model.

It was recently claimed that the superstructure in the stripe phase of a thin film of $\mathrm{La}_{0.5} \mathrm{Ca}_{0.5} \mathrm{MnO}_{3}$ was a prototype CDW with collective transport properties. ${ }^{16}$ This statement was based on the observation of hysteresis in IVCs and broadband noise. However, no clear threshold field or ac voltage generation was observed, which was explained by a large impurity density. Broadband noise observation in manganites was also reported in Refs. 17 and 18. Nonlinear transport properties in manganites were challenged in Ref. 19.

Recently a new class of layered compounds, namely, rare-earth tritellurides $R \mathrm{Te}_{3}(R=\mathrm{Y}, \mathrm{La}, \mathrm{Ce}, \mathrm{Nd}, \mathrm{Sm}, \mathrm{Gd}$, $\mathrm{Tb}, \mathrm{Ho}, \mathrm{Dy}, \mathrm{Er}, \mathrm{Tm})$, has raised an intense research activity on CDWs. ${ }^{20-22}$ These systems exhibit an incommensurate CDW through the whole $R$ series with a wave vector $\mathbf{Q}_{\mathrm{CDW} 1}=\left(0,0, \sim 2 / 7 c^{*}\right)$ with a Peierls transition temperature above $300 \mathrm{~K}$ for the light atoms ( $\mathrm{La}, \mathrm{Ce}, \mathrm{Nd}$ ). For the 
heavier $R$ (Dy, Ho, Er, Tm) a second CDW occurs with the wave vector $\mathbf{Q}_{\mathrm{CDW} 2}=\left(\sim 2 / 7 a^{*}, 0,0\right)$. The superlattice peaks measured from $\mathrm{X}$-ray diffraction are very sharp and indicate a long range three-dimensional (3D) CDW order. ${ }^{22}$

All $R \mathrm{Te}_{3}$ compounds have the same orthorhombic structure $(\mathrm{Cmcm})$ in the unmodulated state. The structure is formed of blocks of $\left[R_{2}{ }^{3+} \mathrm{Te}_{2}{ }^{2-}\right]^{+}$layers sandwiched between the $\mathrm{Te}^{-}$ layers and stacked together along the $b$ axis with a weak van der Waals gap $(\sim 3.8 \AA)$ between them. In the $(a, c)$ plane the Te-Te distances are $3.1 \AA$ (to be compared to the covalent Te-Te bond of $2.8 \AA$ ). The charge transfer from $R$ ions to the Te square yields the $p$ band for Te square sheets to be partially filled. The band structure calculated with a simple tight-binding approximation reveals that the electronic bands of $E_{F}$ derive from $p_{x}$ and $p_{z}$ in-plane Te orbitals, leading to a simple FS. Splitting of the bands occurs due to bilayer Te sheets in the unit cell shifted one with respect to the other by $c / 2$. The hopping between orbitals along a given direction ( $x$ or $z$ ) $t_{\|}$is much larger than the hopping between the same orbitals on neighbor rows $t_{\perp}$. In addition, hopping between second-neighbor $t^{\prime}$ mixes the $p_{x}$ and $p_{z}$ bands. Typically it was estimated to be $t_{\|} \simeq 2.0 \mathrm{eV}, t_{\perp} \simeq 0.37 \mathrm{eV}$, and $t^{\prime} \simeq 0.16 \mathrm{eV}^{23}$ The finite value of $t_{\perp}$ introduces the warping of FS; $t_{\perp}$ is much larger than $300 \mathrm{~K}$, indicating that $1 \mathrm{D}$ approximations are not applicable to $R \mathrm{Te}_{3}$ compounds.

Amplitude CDW excitations in $R \mathrm{Te}_{3}$ were probed by Raman scattering ${ }^{24,25}$ and femtosecond pump-probe spectroscopy. This latter technique has recently allowed to study the disentanglement of the electronic and lattice path of the CDW order parameter, the collective vibrations being assigned to amplitude modes. ${ }^{26}$ Time-resolved angle-resolved photoemisison spectroscopy (ARPES) has also allowed to make collective dynamics of CDW systems after optical excitation to be visible through their influence on the quasiparticle band structure. ${ }^{27,28}$ On the other hand, collective charge phase excitations could not be observed in far-infrared measurements ${ }^{29}$ due to screening by the residual metallic component at the Fermi surface. But the phase collective mode is accessible through nonlinear transport properties, as we report hereafter in $\mathrm{DyTe}_{3}$. It is evidence of $\mathrm{CDW}$ sliding in quasi-2D systems.

$\mathrm{DyTe}_{3}$ was chosen because in this compound the CDW appears just at room temperature at $T_{\mathrm{CDW} 1}=302 \mathrm{~K}$ and the lower CDW at $T_{\mathrm{CDW} 2}=49 \mathrm{~K}$ is the largest in the $R \mathrm{Te}_{3}$ series. The component of the wave vector $\mathbf{Q}_{1}$ along $c^{*}$ was found to be 0.2984 (Ref. 30) (or 0.70 according to whether the 2D Brillouin zone for a single Te plane or the 3D Brillouin zone for the unit cell is considered). This value is slightly far away from $2 / 7$ or $5 / 7$ ( 0.286 or 0.714$)$, which was estimated from band filling resulting from charge transfer between $R$ ions and Te sheets.

A slightly modified method described in Ref. 31 has been used for the growth of the single crystal of $\mathrm{DyTe}_{3}$ : Single crystals were grown by a self-flux technique under purified argon atmosphere in a sealed quartz tube. High quality starting elements were used: $4 \mathrm{~N}$, Te $6 \mathrm{~N}$, and the molar composition of the binary system was $\left(\mathrm{DyTe}_{3}+90 \mathrm{Te}\right)$. The mixture has been heated, in a same run, at 550 and $850{ }^{\circ} \mathrm{C}$, respectively, for 2 days before cooling down with a ramping of $2{ }^{\circ} \mathrm{C} / \mathrm{h}$ to $450{ }^{\circ} \mathrm{C}$ and then quenched in air to room temperature. The

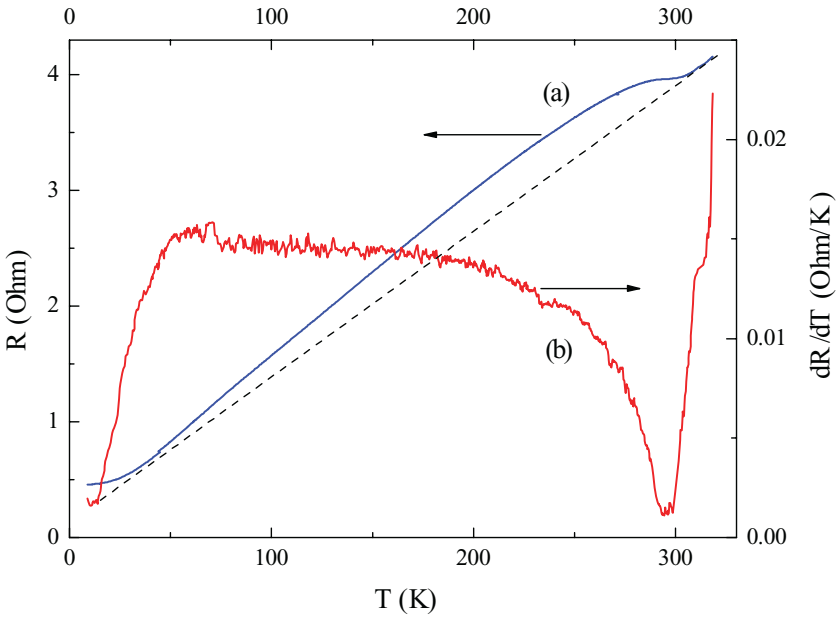

FIG. 1. (Color online) Temperature dependence of resistance [curve (a)] and its derivative [curve (b)] in the $(a-c)$ plane of a $\mathrm{DyTe}_{3}$ single crystal. The dotted line is the linear extrapolation of the resistance from above $T_{\mathrm{CDW} 1}$.

millimeter scale platelike single crystals were mechanically extracted from the bulk. The $b$ axis has been checked to be perpendicular to the large surface of crystals and its value refined $b=25.420 \AA$ for $\mathrm{DyTe}_{3}$.

Thin single-crystal samples were prepared by micromechanical exfoliation of relatively thick crystals glued on a sapphire substrate. From homogeneous square single crystals with thickness $0.4-5.0 \mu \mathrm{m}$, we cut bridges with a width 50-100 $\mu \mathrm{m}$. Measurements of current-voltage characteristics (IVCs) and their derivatives have been performed with a conventional four-probe configuration. The electric field was applied along the $c$-axis direction. Contacts were prepared from In by cold soldering. The distance between potential probes varied in the range $0.2-0.5 \mathrm{~mm}$. For studying nonstationary effects a radio-frequency (rf) current was superposed on the dc current using current contacts connected with the generator via two capacitors.

Figure 1 shows the characteristic $R(T)$ (a) and $d R(T) / d T$ (b) dependencies in the $(a-c)$ plane for one of the $\mathrm{DyTe}_{3}$ samples we measured. The small increase in resistance below $T_{\mathrm{CDW} 1}=302 \mathrm{~K}$ is the signature of the Peierls transition in this compound. On the $d R(T) / d T$ this effect is much more pronounced. Our data in Fig. 1 are very similar to those in Ref. 22. No visible variation in the resistance was detectable for the CDW transition at $T_{\mathrm{CDW} 2}$.

In Fig. 2 we have drawn the differential IVCs in the temperature range $195<T<301 \mathrm{~K}$ for a sample with a thickness of $0.5 \mu \mathrm{m}$. A pronounced nonlinearity is observed below $300 \mathrm{~K}$. Although the amplitude of this nonlinearity is small $\left(\triangle R / R \sim 10^{-2}\right)$, the threshold behavior typical for the transition to the sliding CDW state is clearly seen: The IVCs are ohmic for a voltage that is less than some threshold voltage $V_{T}$, and for voltages in excess of this value the differential resistance, $R_{d}=d V / d I$, decreases sharply. At temperatures $T>T_{P}=302 \mathrm{~K}$ the IVCs are ohmic without any nonlinearities, except for very little Joule heating which appears as a small parabolic increase of the differential 


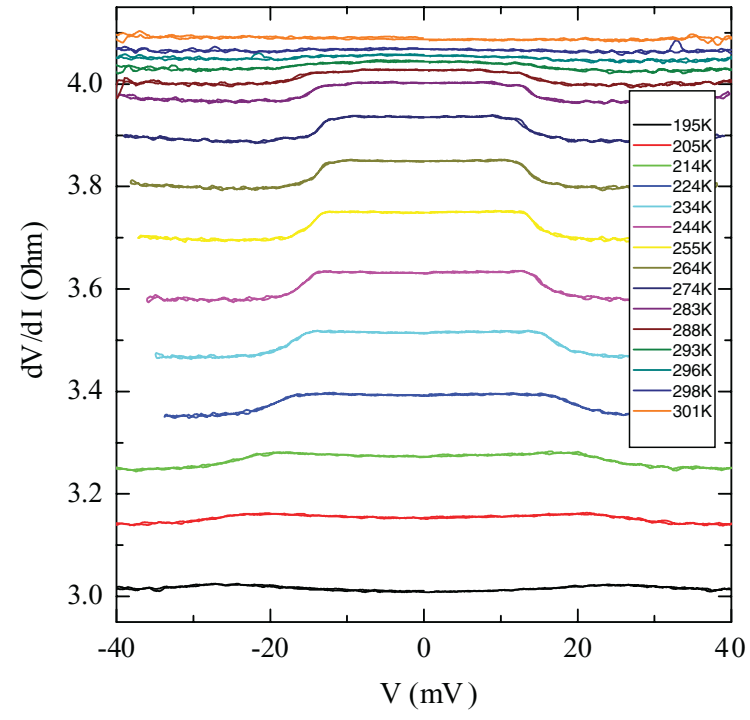

FIG. 2. (Color online) Differential resistance $R_{d}=d V / d I$ as a function of the applied voltage $V$ at different temperatures varying from 195 to $301 \mathrm{~K}$ for a $\mathrm{DyTe}_{3}$ single crystal with a thickness $0.5 \mu \mathrm{m}$.

resistance. For thicker samples, the threshold behavior is smeared by Joule heating.

The very sharp onset of the observed nonlinearity allows to determine the temperature dependence of the threshold electric field $E_{T}$, which is shown in Fig. 3 . As can be seen, $E_{T}$ initially decreases in the temperature range 301-265 $\mathrm{K}$ and increases when the temperature is decreased further. Note that such a behavior is typical for $E_{T}$ in quasi-one-dimensional systems with a CDW. ${ }^{1}$

It is well known that the joint application of dc and rf driving fields leads to the appearance of harmonic and subharmonic Shapiro steps in the dc IVCs of 1D CDWs. ${ }^{1}$ In the present work

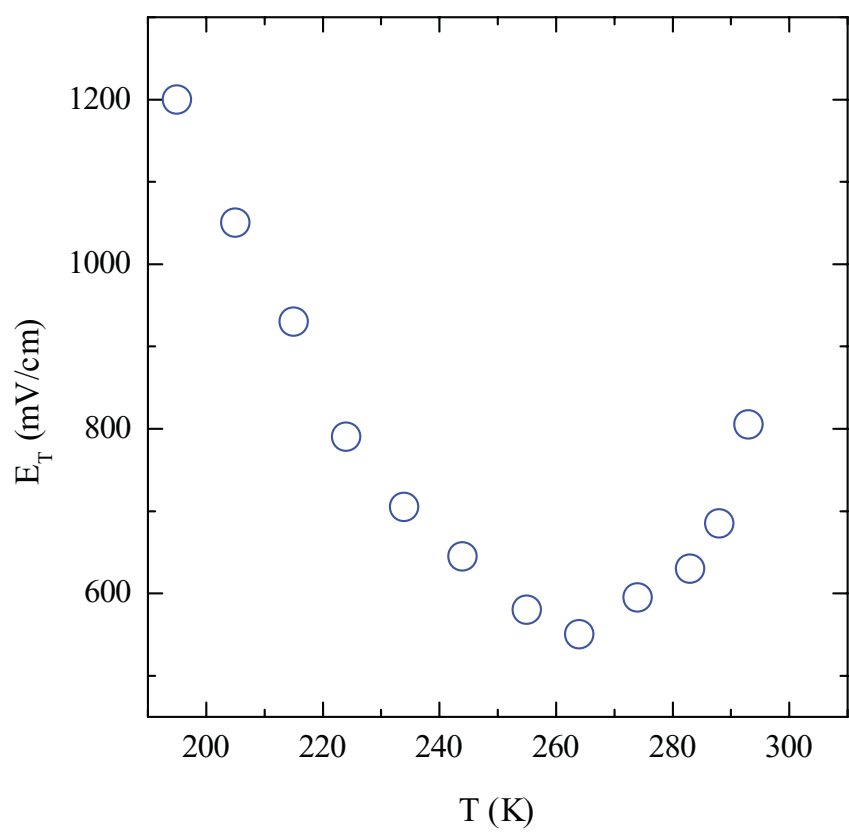

FIG. 3. (Color online) Temperature dependence of the threshold field $E_{T}$ for the same sample as shown in Fig. 2.

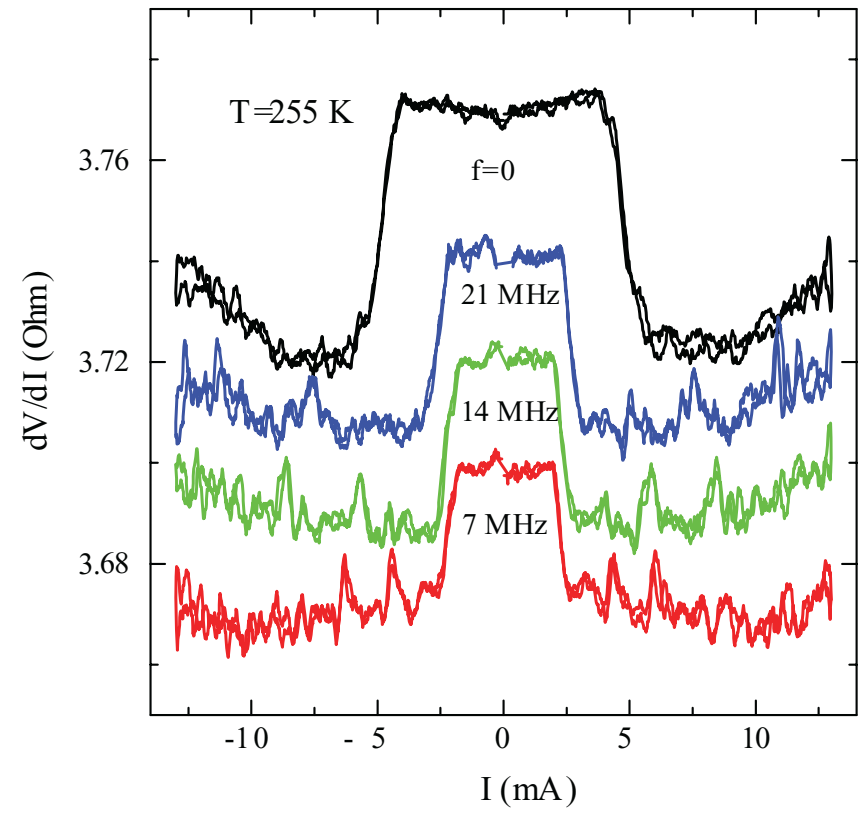

FIG. 4. (Color online) $d V / d I(I)$ dependencies at $T=255 \mathrm{~K}$ under application of a rf field with a given frequency 7, 14, and $21 \mathrm{MHz}$ for the same sample as shown in Fig. 2. For comparison, the static (without rf field) differential curve is also shown. The curves are shifted relative to each other for clarity.

we have also observed Shapiro steps at such experimental conditions. Figure 4 shows $d V / d I(I)$ dependencies at $T=$ $255 \mathrm{~K}$ under application of a rf field with a frequency of 7 , 14 , and $21 \mathrm{MHz}$ with the same rf power. For comparison, the static (without rf field) differential IVC measured at this temperature is also shown. The curves are shifted relative to each over for clarity. First of all, note that application of a rf electric field leads to a reduction in the threshold electric field $E_{T}$. At the same time, Shapiro steps appear in the $d V / d I(I)$ characteristic as sharp maxima in the differential resistance. With increasing frequency the distance between neighboring maxima increases proportionally to the frequency.

The observation of Shapiro steps is one of the characteristic features of a CDW collective transport. ${ }^{1}$ Thus, the observed nonlinearities of dc IVCs, together with the observation of Shapiro steps, evidently indicates that a contribution to the electrical transport from the collective CDW motion takes place in the 2D CDW DyTe 3 compound.

It is worth noting that the effect of CDW on the resistance in the $(a-c)$ plane is very weak (see Fig. 1) and consists of only a few percent increase below $T_{\mathrm{CDW} 1}$. In fact, the larger effect is along the $b$ axis, perpendicular to the Te sheets. ${ }^{22} \mathrm{It}$ is commonly explained that the reduction of the Fermi surface (FS) by opening of the CDW gap reduces the scattering of electrons and therefore the conductivity. From ARPES and optical studies it was shown that at low temperatures about $30-40 \%$ of the FS in $\mathrm{ErTe}_{3}$ and $\mathrm{HoTe}_{3}$ is affected by the formation of the CDW. ${ }^{32}$ The small decrease of conductivity below $T_{\mathrm{CDW} 1}$ may indicate that electrical transport does not significantly involve bands on which the CDWs occur.

Although the temperature dependence of the gap amplitude was found to follow a BCS type, the ratio $2 \Delta / k_{B} T_{\mathrm{CDW}} \simeq$ $15-17$ is much larger than the mean field 3.52 value. Such 
a large magnitude was already measured in transition metal dichalcogenides, and specifically in $2 \mathrm{H}-\mathrm{TaSe}_{2}$. That led McMillan to develop a strong coupling model for systems with a short coherence length where phonon frequencies are modified over large parts of reciprocal space. ${ }^{33}$

The crystallographic analysis gave for $R \mathrm{Te}_{3}$ a distribution of Te-Te bond lengths in the Te nets, ${ }^{30,34,35}$ such as a minimum distance $2.9564 \AA$ and a maximum $3.2407 \AA$ in $\mathrm{CeTe}_{3}$. Thus, the Te net was viewed as an oligomer sequence of trimers and tetramers. Such a discommensurate nature of the CDW (Ref. 36) in scanning tunneling microscopy (STM) data in $\mathrm{CeTe}_{3}$ was challenged in Ref. 37, which established a fully incommensurate $\mathrm{CDW}$ in $\mathrm{TbTe}_{3}$. However, the CDW has a strong effect on the lattice. In $\mathrm{TbTe}_{3}$ it was shown ${ }^{22}$ that the formation of the CDW stretched the lattice along the direction of the modulation wave vector ( $c$ axis) in the sense that the $T$ dependence of the $c$ parameter below $T_{\mathrm{CDW}}$ is much less than its extrapolation from above $T_{\mathrm{CDW}}$, at variance with the $T$ dependence of the $a$ parameter that may indicate a strong electron-phonon coupling. It would be worth to perform very high sensitivity temperature dependence of the $\mathbf{Q}$ vector in order to firmly establish if thus $T$ dependence is smooth or if small discontinuities are present: those being induced by a charge redistribution followed with a Te-Te bond length change. This process would result from the flexibility of telluride to exhibit a wide range of Te-Te bonds. ${ }^{30}$

In conclusion, we have shown that the phase of the $3 \mathrm{D}$ long range order $\mathrm{CDW}$ in the layered $\mathrm{DyTe}_{3}$ compound can easily slide above a small threshold field. Thus we demonstrate CDW sliding in two-dimensional compounds. It has been noted that the physical properties of $R \mathrm{Te}_{3}$ compounds are essentially determined by the Te planes. Even if correlations in $R \mathrm{Te}_{3}$ are smaller than in cuprates, some analogy may be reasonably made, specifically with respect to the nature of the order parameter of the pseudogap state in HTSC for which the broken symmetry may have a smectic and/or a nematic character. ${ }^{10}$ The semidirectional density wave in rare-earth tellurides may correspond to a static stripe order. The occurrence of two orthogonal CDWs with nearly identical wave vectors in the case of the heaviest rare earth, which may lead to a bidirectional checkboard superstructure, is very compelling. ${ }^{23}$

The authors are thankful to J. Marcus for help in the sample preparation. The work has been supported by Russian State Fund for the Basic Research (No. 11-02-01379), and partially performed in the frame of the CNRS-RAS Associated International Laboratory between Institut Néel and IRE "Physical properties of coherent electronic states in coherent matter."
${ }^{1}$ G. Grüner, Density Waves in Solids (Addison-Wesley, Reading, MA, 1994); L. Gor'kov and G. Grüner, Charge Density Waves in Solids (Elsevier Science, Amsterdam, 1989); Electronic Crystals 2011, edited by S. Brazovskii, P. Monceau, and N. Kirova, Physica B 407, 1683 (2012).

${ }^{2}$ A. W. Overhauser, Phys. Rev. 128, 1437 (1962).

${ }^{3}$ W. M. Lomer, Proc. Phys. Soc. 80, 489 (1962).

${ }^{4}$ E. Fawcett, Rev. Mod. Phys. 60, 209 (1988).

${ }^{5}$ E. Fawcett, H. L. Alberts, V. Yu. Galkin, D. R. Noakes, and J. V. Yakhmi, Rev. Mod. Phys. 66, 25 (1994).

${ }^{6}$ H. Fröhlich, Proc. R. Soc. London, Ser. A 223, 296 (1954).

${ }^{7}$ P. Monceau, Adv. Phys. (2012) (to be published).

${ }^{8}$ H. Seo, J. Merino, H. Yoshioka, and M. Ogata, J. Phys. Soc. Jpn. 75, 051009 (2006).

${ }^{9}$ E. Dagotto, T. Hotta, and A. Moreo, Phys. Rep. 344, 1 (2001).

${ }^{10}$ M. Vojta, Adv. Phys. 58, 699 (2009).

${ }^{11}$ F. J. DiSalvo and R. M. Fleming, Solid State Commun. 35, 685 (1980)

${ }^{12}$ V. J. Goldman, M. Santos, M. Shayegan, and J. E. Cunningham, Phys. Rev. Lett. 65, 2189 (1990).

${ }^{13}$ Y. P. Chen, R. M. Lewis, L. W. Engel, D. C. Tsui, P. D. Ye, L. N. Pfeiffer, and K. W. West, Phys. Rev. Lett. 91, 016801 (2003).

${ }^{14}$ G. Sambandamurthy, R. M. Lewis, Han Zhu, Y. P. Chen, L. W. Engel, D. C. Tsui, L. N. Pfeiffer, and K. W. West, Phys. Rev. Lett. 100, 256801 (2008).

${ }^{15}$ K. B. Cooper, J. P. Eisenstein, L. N. Pfeiffer, and K. W. West, Phys. Rev. Lett. 90, 226803 (2003).

${ }^{16}$ S. Cox, J. Singleton, R. D. McDonald, A. Migliori, and P. B. Littlewood, Nat. Mater. 7, 25 (2008).

${ }^{17}$ A. Wahl, S. Mercone, A. Pautrat, M. Pollet, Ch. Simon, and D. Sedmidubsky, Phys. Rev. B 68, 094429 (2003).
${ }^{18}$ C. Barone, A. Galdi, N. Lampis, L. Maritato, F. M. Granozio, S. Pagano, P. Perna, M. Radovic, and U. Scotti di Uccio, Phys. Rev. B 80, 115128 (2009).

${ }^{19}$ B. Fisher, J. Genossar, L. Patlagan, S. Kar-Narayan, X. Moya, D. Sánchez, P. A. Midgley, and N. D. Mathur, J. Phys.: Condens. Matter 22, 275602 (2010).

${ }^{20}$ E. DiMasi, M. C. Aronson, J. F. Mansfield, B. Foran, and S. Lee, Phys. Rev. B 52, 14516 (1995).

${ }^{21}$ V. Brouet, W. L. Yang, X. J. Zhou, Z. Hussain, R. G. Moore, R. He, D. H. Lu, Z. X. Shen, J. Laverock, S. B. Dugdale, N. Ru, and I. R. Fisher, Phys. Rev. B 77, 235104 (2008).

${ }^{22}$ N. Ru, C. L. Condron, G. Y. Margulis, K. Y. Shin, J. Laverock, S. B. Dugdale, M. F. Toney, and I. R. Fisher, Phys. Rev. B 77, 035114 (2008).

${ }^{23}$ H. Yao, J. A. Robertson, E.-A. Kim, and S. A. Kivelson, Phys. Rev. B 74, 245126 (2006).

${ }^{24}$ M. Lavagnini, H. M. Eiter, L. Tassini, B. Muschler, R. Hackl, R. Monnier, J. H. Chu, I. R. Fisher, and L. Degiorgi, Phys. Rev. B 81, 081101(R) (2010).

${ }^{25}$ M. Lavagnini, M. Baldini, A. Sacchetti, D. DiCastro, B. Delley, R. Monnier, J. H. Chu, N. Ru, I. R. Fisher, P. Postorino, and L. Degiorgi, Phys. Rev. B 78, 201101 (2008).

${ }^{26}$ A. Tomeljak, H. Schafer, D. Stadter, M. Beyer, K. Biljakovic, and J. Demsar, Phys. Rev. Lett. 102, 066404 (2009).

${ }^{27}$ F. Schmitt, P. S. Kirchmann, U. Bovensiepen, R. G. Moore, L. Rettig, M. Krenz, J.-H. Chu, N. Ru, L. Perfetti, D. H. Lu, M. Wolf, I. R. Fisher, and Z.-X. Shen, Science 321, 1649 (2008).

${ }^{28}$ F. Schmitt, P. S. Kirchmann, U. Bovensiepen, R. G. Moore, J.-H. Chu, D. H. Lu, L. Rettig, M. Wolf, I. R. Fisher, and Z.-X. Shen, New J. Phys. 13, 063022 (2011). 
${ }^{29}$ M. Lavagnini, A. Sacchetti, C. Marini, M. Valentini, R. Sopracase, A. Perucchi, P. Postorino, S. Lupi, J.-H. Chu, I. R. Fisher, and L. Degiorgi, Phys. Rev. B 79, 075117 (2009).

${ }^{30}$ C. D. Malliakas, Dissertation, Michigan State University, 2008.

${ }^{31}$ N. Ru and I. R. Fisher, Phys. Rev. B 73, 033101 (2006).

${ }^{32}$ F. Pfuner, P. Lerch, J.-H. Chu, H.-H. Kuo, I. R. Fisher, and L. Degiorgi, Phys. Rev. B 81, 195110 (2010).

${ }^{33}$ W. L. McMillan, Phys. Rev. B 16, 643 (1977).
${ }^{34}$ C. D. Malliakas, S. J. L. Billinge, H. J. Kim, and M. G. Kanatzidis, J. Am. Chem. Soc. 127, 6510 (2005).

${ }^{35}$ C. D. Malliakas and M. G. Kanatzidis, J. Am. Chem. Soc. 128, 12612 (2006).

${ }^{36}$ H. J. Kim, C. D. Malliakas, A. T. Tomic, S. H. Tessmer, M. G. Kanatzidis, and S. J. L. Billinge, Phys. Rev. Lett. 96, 226401 (2006).

${ }^{37}$ A. Fang, N. Ru, I. R. Fisher, and A. Kapitulnik, Phys. Rev. Lett. 99, 046401 (2007). 